\title{
Promoting Sustainability Culture among Citizens: A System Dynamic Analysis of the Role of Schools
}

\author{
ELENA Claire RicCI \\ elenaclaire.ricci@univr.it \\ University of Verona
}

\begin{abstract}
This paper concerns the issue of how to promote sustainable behaviours among citizens and consumers, that is increasingly viewed as crucial to tackle global social and environmental problems. More in detail, it investigates the potential role of schools at all levels, from kinder garden to university, in their relation to (mainly) local stakeholders. The study aims at shedding light on potential diffusion dynamics of sustainable behaviour among individuals when schools, universities and other stakeholders are involved. To do so, we develop a simple system dynamics model, that is an approach that is well suited to model non-linear dynamics, interdependencies, and time lags typical of human decision processes and behaviour. The model proposed is a first preliminary attempt to highlight the system effects that could be triggered by active sustainability projects in schools. The variables included in the model and the data are taken from an action-research project 'Sustainability Labs' that is being carried out in a set of schools in the Milan (Italy) area. Results suggest that taking advantage of the schooluniversity-local stakeholder triad is able to reach many people and in quite an engaging way that is likely to have interesting results in terms of behavioural change.
\end{abstract}

\section{Introduction}

Literature is increasingly showing the importance of engaging with citizens and consumers to identify and carryout possible solutions to global societal and environmental challenges (Steg and Vlek, 2009; Ricci and Banterle, 2018). Citizens/consumers are becoming increasingly attentive to sustainability issues (European Commission, 2014) and accustomed to taking an active role in many sectors in the emerging smart cities and communities. Moreover, the recent aggregation induced by the initiatives led by the young climate activist Greta Thunberg and the Fridays for Future movement have shown how citizens - especially the young - are willing to be engaged on sustainability matters.

Many studies have investigated what types of consumers follow sustainability related principles while choosing among products, providers and behavioural options. Much of the literature identifies socio-demographic characteristics as crucial factors in affecting consumer behaviour, highlighting the role of gender, income and education levels (Drichoutis, Lazaridis and Nayga Jr, 2006; Pedersen and Neergaard, 2006; D'Souza et al., 2007; Testa et al., 2015). However, most of the studies focus on types of behaviour that require additional spending for 'sustainable choices'. In many applications, though, sustainability behaviours go in the same direction as money saving, thus enlarging the lens of modelled behaviours might weaken the 
assumption that - ceteris paribus - mainly female, educated, and rich people are engageable in 'sustainable' behaviours. This is important if sustainability behaviour is to get out the niche and become mainstream.

Other studies highlight the role of other variables related to personal attitudes and values in affecting sustainable behaviour (Ajzen, 1991; Vermeir and Verbeke, 2006; Arvola et al., 2008; Agovino et al., 2017). Such inclinations depend on the single individual and, although possibly related to some socio-demographic variables, they vary within socio-demographic segments. We here refer to people as individuals that are both citizens (that are able to vote, that decide on their social behaviour and participation) and consumers (that choose what products and services to buy). Concern and knowledge about sustainability has also been identified as a relevant factor in directing pro-social and pro-environmental behaviour (De Groot and Steg, 2007; Hartmann and Apaolaza-Ibáñez, 2012; Urban and Ščasný, 2012).

Variables related to personal norms, social norms and knowledge are long-lived and not easily changed, especially in the short term. While information can be provided, building up this information so that it can become knowledge and then be integrated in one own's culture it is a complex and dynamic process. Indeed, many studies show that information provision is helpful, but it is not a quick fix to align people's behaviour to the social optimal (Eden, Bear and Walker, 2008). Especially in certain domains, like food and energy use, that entails many decisions many times per day; thus promoting behavioural changes implies changing habits, which involve both cognitive and somewhat-automated behaviours (Verplanken and Aarts, 1999; Daniels et al., 2015; Kurz et al., 2015). Human decision processes are very complex and need to be studied with models fit to get the 'systemic' picture and to take into account the non-linearities of the processes involved (Ricci, 2013).

If affecting individual values and behaviour is a complex and uncertain process, trying to influence social norms is an even more ambitious aim. These are the outcome of prolonged interactions among individuals and are strongly related to the cultural, social, environmental and political, etc., context in which these interactions take place. However, sometimes unexpected events might suddenly increase the sensitivity of people and generate sudden shifts in behaviour. An example of such types of events is related for example to the recently triggered debate and active citizenship about climate change.

The 'Greta Effect' further highlights the reasons for building systemic and dynamic models that are able to account for sudden shifts in behavioural dynamics triggered, for example, by an increase in saliency of specific issues brought about by the interaction among different and new actors. This is particularly important when focusing on sustainable development. Indeed, the goal of sustainability opens to new scenarios, new dimensions in play, new dynamics, and even new actors. Thus, it requires to at least reconsider and re-evaluate current assessment models, to verify if they are sensitive enough to be able to identify new emerging dynamics. For example, events like the 'Greta effect' cannot be captured by studying the spread of "sustainable behaviour" as if it was just any other (less ethical and engaging) behaviour and/or just looking at past data. Sustainability research needs systemic, non-linear, and flexible models to attempt to capture system complexity.

This paper attempts to go in this direction highlighting the potential role of schools, at all levels, in conjunction with other 'glocal' (local and global) stakeholders in promoting the diffusion of sustainability culture and, in attempting, to affect citizen/consumer behaviour. The aim is to simulate scenarios about potential diffusion dynamics of acknowledged changes 
in decision making processes (here applied to everyday food and energy choices) to include additional variables related to sustainability. More in detail, this implies considering not only traditional variables like costs and/or taste, but also the environmental, social, organisational, health, and/or cultural effects (and much more) of the different options. The system dynamics (Forrester, 1961; Sterman, 2000) model proposed is a first preliminary attempt to highlight the system effects that could be triggered by active sustainability projects in schools. The variables included in the model and the data are taken from an action-research project 'Sustainability Labs' that is being carried out in a set of schools in the Milan (Italy) area. The scope of the paper is mainly qualitative and methodological to show, on one side, the potential effects of actively engaging with schools and, on the other, suggest the use of system dynamics models to study the dynamics of complex phenomena.

In this perspective, schools and universities constitute an interesting focus, particularly suitable for developing and testing the new organisational models linked to sustainability, in the emerging ' knowledge society', that is, a highly information-intensive society characterised by high levels of interaction between different stakeholders and one that empowers the knowledge of the actors involved. In particular, schools and universities constitute primary vectors of knowledge and culture diffusion (Rieckmann, 2012; Lozano et al., 2013) and have a fundamental role in promoting the 'profound change' needed to respond to global challenges that today's societies are facing (Kühtz, 2007). In fact, they can reach a large number of people, especially young people, who will play an important role in the transition to a more sustainable society, both as citizens and as professionals (Bremer and López-Franco, 2006; Sibbel, 2009). Moreover, recent studies highlight how many behavioural habits that entail different sustainability impacts, like electricity use and food choices, are set at a very early age (Aguirre-Bielschowsky et al., 2018).

\section{Sustainable consumer behaviour}

Most studies find that the so-called 'green consumer' is (ceteris paribus) - more likely - to be young, female, with a high level of education and income, and with children (Gilg, Barr and Ford, 2005; Pedersen and Neergaard, 2006; Boccaletti, 2008). However, other studies advocate that green consumption is also related to environmental and social values (Gilg, Barr and Ford, 2005). A paper by Vermeir and Verbeke (2008) suggests that half of variability in the intention for sustainable consumption is 'explained by personal attitudes, perceived social influences, perceived consumer effectiveness, and perceived availability'. Moreover, others associate sustainable consumption with 'good parenting' and 'ecological citizenship' (Evans, 2011).

This research takes this second stance in considering sustainable behaviour strongly related to personal and social norms and values; and aims at investigating what could be the potential role of schools, university and other stakeholders in engaging individuals and impacting their decision-making processes.

In fact, from the literature it emerges that although there is interest and concern for environmental problems, these often do not translate into changes in the lifestyles or consumption of citizens (Vermeir and Verbeke, 2006; Galarraga et al., 2011). Many studies have investigated the possible reasons behind this identifying several issues.

One of the main barriers that do not allow the interest in sustainability to turn into action is related to lack of knowledge. This shortage includes little knowledge about sustainability in 
general, its multi-dimensional and multi-disciplinary integrated dimensions (or pillars), and also the difficulty to reason in inter-temporal terms. Moreover, literature suggests scarcity of knowledge regarding the impacts of one's choices (Kahan et al., 2011; Pongiglione, 2012). This is related to perceived consumer effectiveness (PCE) and environmental responsibility attribution (Gilg, Barr and Ford, 2005). Indeed, the important role of feedback about the positive and negative impacts of (one's own) previous choices has been highlighted (Darby, 2006; Darby, 2010). More recent literature also suggests that comparison with the behaviour of others might also trigger behavioural changes (Thaler and Sunstein, 2008; Alcott, 2009; Sunstein, 2013).

Sometimes, given the low knowledge about sustainability issues and the impact of one's own choices, there is a lack of procedural knowledge regarding how to actually take more sustainable decisions (Pongiglione and Cherlet, 2015).

The hypothesis of this research is that the spread of a culture of sustainability (in partnership between schools and universities) and the development of concrete initiatives organised in collaboration with schools, universities, (g)local communities and companies can lead to a greater involvement of individuals on the topic and promote the diffusion of sustainable behaviour by integrating a greater set of variables into decision making processes, which can then lead to spillovers in many different domains. However, it must be noted that actual behavioural changes are not only related to intentions to perform a behaviour, but also to the availability of sustainable options (Ajzen, 2011). Indeed, the viscosity of the economic system and the refractoriness of some economic agents in moving towards more sustainability pathways can hinder behavioural changes. Indeed, in some cases sustainable behaviours are not incentivised, like when sustainability options are, for example, limited, more expensive, difficult to acquire or to identify, or they require more effort and time by users, and so on.

\section{Methods}

\subsection{Stakeholder engagement}

The idea of setting up a project aimed at engaging with schools on sustainability matters came for the consideration, that at least in Italy, schools have been lagging behind other private and public actors in promoting sustainability. Up until 2015, when the Sustainability Project started, and thereafter no official state school program (excluding university courses) included topics of sustainability or sustainable development. Moreover, hardly any specific school program documents - that are developed and published each year by each school - included the word sustainable development or sustainability. The only topic included, in the programs of some schools, was related to so called environmental education. However, sustainability is much more than this. In 2019, for the first time, official documents of the Education Ministry indicate that these topics should be discussed, even if they do not provide specific guidelines as they instead do for other topics.

However, schools could be very effective vectors of diffusion of sustainability culture. In fact, on the Italian territory there are more than 72,500 (Istat Data) schools that can be involved on an ambitious ethical goal of sustainability culture diffusion and possibly of thus inducing more sustainable choices and practices. 
The 'Sustainability Labs' project aims at identifying and testing ways of creating a distributed culture about sustainability in an open manner engaging with schools, (g)local communities, socio-economic actors and all possible stakeholders. The aim being to diffuse systemic reasoning and the inclusion of $360^{\circ}$ sustainability evaluations in decision making processes of individuals (and all possible actors). The tools proposed aim at enhancing the cultural and social responsibility of schools and universities, and their ability to aggregate people and trigger changes in consumption patterns, and at analysing how 'glocal' resources (financial, organisational, environmental, socio-cultural) can be boosted to promote practical sustainability projects and their multi-level spillovers. Indeed, the main focus of the project is to build a partnership between universities and schools so that the former may support the latter in building sustainability projects, that should include students, their families, the local (and global) community.

The idea is to promote a participatory and interactive approach where there is no unique strategy imposed but initiatives are devised with the schools and communities and supported by the knowledge and competences of universities. Moreover, the approach is also 'glocal' as it aims at integrates self-organised initiatives - that take advantage of local and global opportunities and resources - in an interactive network where experiences, ideas, suggestions, initiatives and funding schemes may be shared, with the aim of identifying successful pathways for the promotion of sustainability.

The project involves schools at different levels (from kinder garden to university level). During the project, several types of qualitative and quantitative data were collected via discussion with headmasters, teachers and via the acquired practice in trying to propose and promote sustainability related projects. Moreover, surveys are administrated each year to both students (while at school) and to parents, collecting - among other information - data about the exchange of information between the students and the parents about the sustainability initiatives and topics, and also about the effects of such information and discussion on family behaviour and choices specifically related to electricity use and food choices in the context of climate change.

\subsection{Scenario analysis}

To analyse the possible dynamics of involvement and interaction of the triad user-providerstakeholder we propose the use of system dynamics simulation models (Forrester, 1961), particularly suitable for studying phenomena in which the human factor is relevant as they pay attention to non-linear interactions between the variables, to internal feedback and to effects delays, typical of the development of knowledge and the adoption of behaviours. The proposed model is constructed starting from the diffusion models of technologies (Bass, 1969; Sterman, 2000) and epidemics (Murray, 2002).

First of all, we created a map representing the relationships among the variables that can influence the processes involved. This essentially qualitative phase often constitutes an opportunity for in-depth analysis (Meadows, 2008) and the resulting map can already itself be used for discussion about the phenomena. Because of the amount of variables often included in the models and the complex net of interdependencies, the second step of the modelling involves the construction of a computer-based numerical model to assess quantitatively and graphically the emerging dynamics (Forrester, 2007; Mella, 2012). 
More specifically, we developed the map on the basis of the outcomes of a few years of implementation of the Sustainability Labs project and discussion with school actors. Then the conceptual map was developed into a model defining the quantitative specification of the relationships between variables and the simulation of temporal dynamics. More in detail, given the limited availability of data to calibrate the model parameters, we apply a Monte Carlo approach simulating 1,000 parameter combinations drawn by probability distributions of the uncertain parameters taken either from the literature or from the results of our data collection with student parents. More in detail, since 2016 we have collected data with a panel of 417 families of two schools in Milan, Italy. Each year we carry out a questionnaire asking families different sets of questions regarding sustainability: if the children have discussed the sustainability-related activities performed at schools; if this discussion has triggered some kind of behavioural change; their general attitude towards sustainability; the perceived issues in teaching sustainability to their children; and a set of questions related to sustainability behaviours and barriers.

Simulations have been performed using Vensim ${ }^{\mathrm{TM}}$ PLE Plus, version 5.11 (Eberlein and Peterson, 1992).

\section{Modelling the diffusion of behavioural changes}

The model built to analyse the potential diffusion of sustainability awareness, culture and consequent behavioural change builds on Bass (1969) and on epidemiological models used in mathematical biology (Murray, 2002). Indeed, we divide families in 'susceptible' and 'infected' as it is done in Susceptible-Infectious (SI) models used to describe the evolution of epidemics in epidemiology. In our model, the 'disease' is behavioural change following an increased sustainability awareness and culture. We also take inspiration from the Bass model on the diffusion of products. This model highlights the interactions between individuals (divided into 'users' and 'potential users') highlighting the role of word-of-mouth and also includes other external information sources. While word of mouth is considered in the marketing literature, to our knowledge, it is not so common to evaluate the spread of ideas, culture or norms by means of this kind of epidemiological-based studies.

The model proposed here is only a first explorative example as it is based on limited and hypothetical data. In fact, equations and figures are reported in general form to indicate how the entire simulation structure can be further customised and applied to different and integrated levels: national, regional or local, focusing on specific areas, particular districts and cities.

Figure 4.1 shows the simulation model: the double arrows with valve show the flows of Schools and Families among the 'stocks' identified by the boxes. The blue arrows identify the processes of influencing these flows. For this first application we bound the model to the families that have children in school age. 


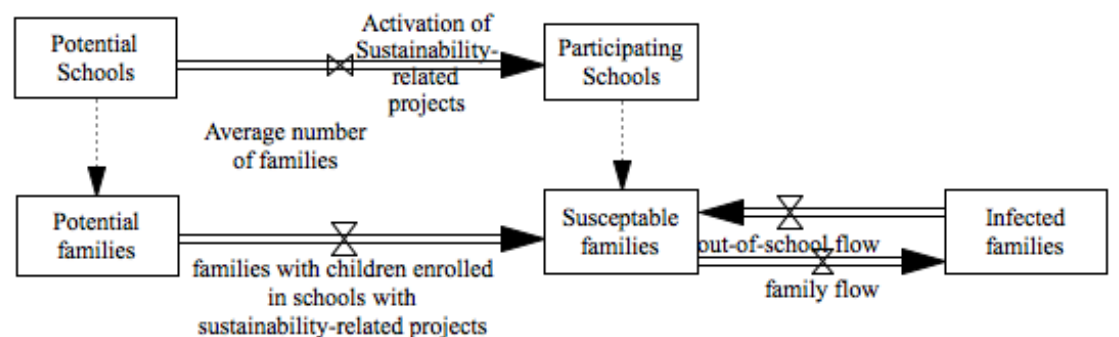

Figure 4.1. The stock - flow structure of the model.

Indeed, the main structure of the model is a system of five non-linear first order finitedifference equations (Eq. 1) that model the integrated evolution of the different states of the two sets of actors included in the model; the variables and their abbreviations are listed in Table 4.1. A further level concerning single individuals could be added to model; however, given that many sustainability decisions are taken at the household level, in this first model we stop at the family level.

$$
\left\{\begin{array}{c}
\operatorname{PoS}_{t+1}-\operatorname{PoS}_{t}=-\operatorname{as}\left(\operatorname{PaS}_{t}, I F_{t}\right) \\
\operatorname{PaS}_{t+1}-\operatorname{PaS}_{t}=+\operatorname{as}\left(\operatorname{PoS}_{t}, I F_{t}\right) \\
\operatorname{PF}_{t+1}-\operatorname{PF}_{t}=-\boldsymbol{f p s}\left(\operatorname{PoS}_{t}, I F_{t}\right) \\
S F_{t+1}-S F_{t}=+\boldsymbol{f p S}\left(\operatorname{PoS}_{t}, I F_{t}\right)-f f\left(\operatorname{PaS}_{t}, S F_{t}, I F_{t}\right)+\operatorname{osf}_{t}\left(\operatorname{PaS}_{t}, S F_{t}, I F_{t}\right) \\
I F_{t+1}-I F_{t}=+f f\left(\operatorname{PaS}_{t}, S F_{t}, I F_{t}\right)-\operatorname{osf}\left(\operatorname{PaS}_{t}, S F_{t}, I F_{t}\right)
\end{array}\right.
$$

\begin{tabular}{|c|c|c|}
\hline Variable & Short name & Type of variable \\
\hline Potential Schools & PoS & Stock of schools \\
\hline Participating Schools & PaS & Stock of schools \\
\hline Potential Families & PF & Stock of families \\
\hline Susceptible Families & SF & Stock of families \\
\hline $\begin{array}{c}\text { Infected Families } \\
\text { Activation of sustainability-related } \\
\text { projects in schools }\end{array}$ & IF & Stock of families \\
\hline $\begin{array}{c}\text { Families with children enrolled in schools } \\
\text { with sustainability-related projects }\end{array}$ & fps & Flow of schools \\
\hline Family Flow & $f f$ & Flow of families \\
\hline Out-of-school family flow & osf & Flow of families \\
\hline
\end{tabular}

Table 4.1. Table of stock and flow variables.

More in detail, the first part of the model regards schools and the potential dynamics of involvement in active sustainability related projects. This part of the model is the one that relies on more hypothetical data, given that the Sustainability Labs projects is currently working only with a set of schools in the Milan area.

Each school involved has a potential pool of families to be invited to participate in the initiative ('susceptible families'), who can take an active part in the project and be potentially involved in changing, at least partially, some behaviours. 
We also assume that every family that has been 'infected', that is, made more sensitive to the topic, can decide to change their behaviour.

Figure 4.2 highlights all the variables included in the fully specified model. Return feedback symbols indicate the reinforcing effects of imitation and word-of mouth that is triggered as participants increase. All exogenous parameters reported in Figure 4.2 have been included in the sensitivity analysis. 


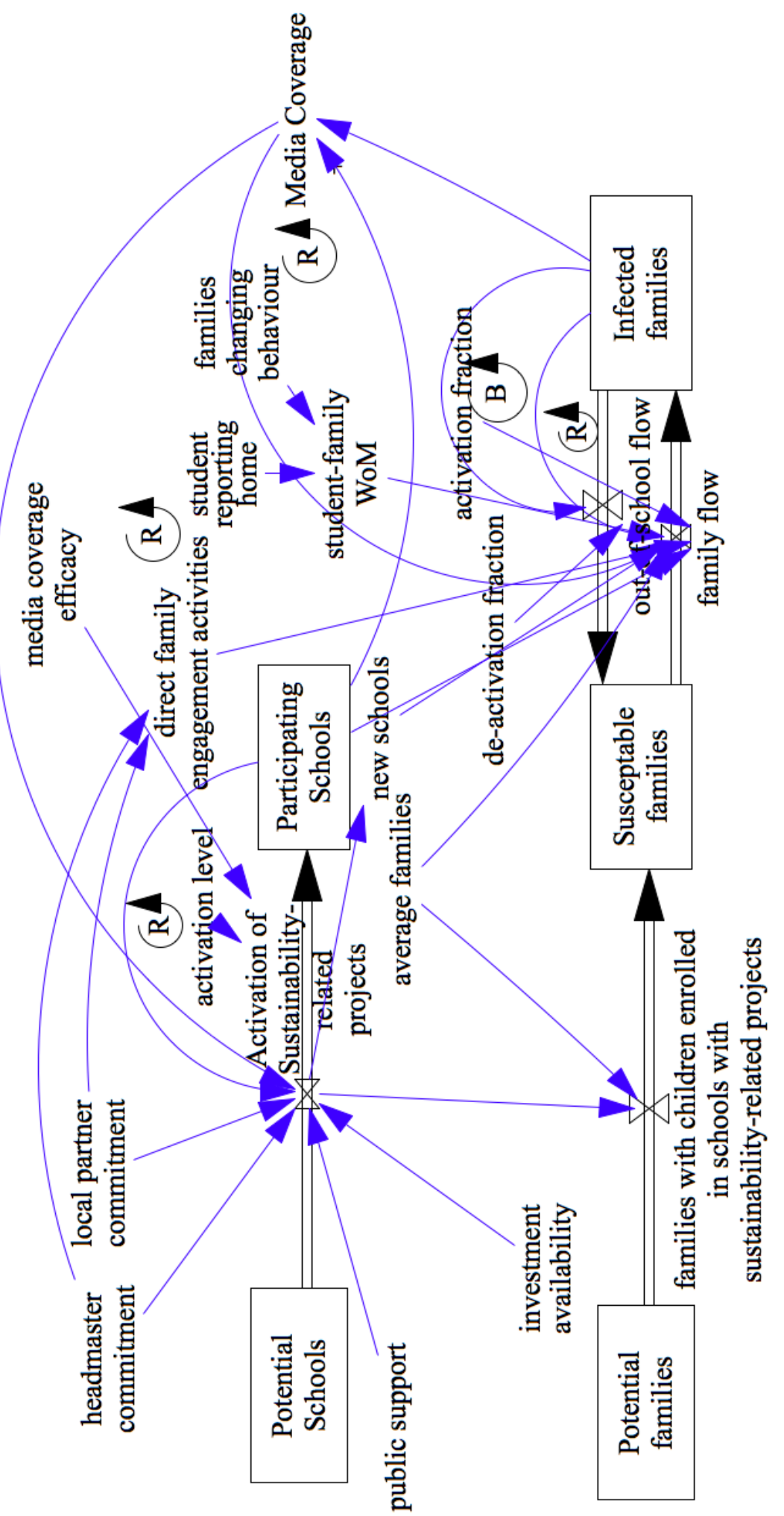

Figure 4.2. The simulation model. 
Even if we do not model it here - due to difficulties in calibration - the model could be further extended to include the potential of "contagion" beyond the families that orbit around the engaged schools by sharing experiences (via word of mouth) and/or media coverage of the initiative. Figure 4.3 reports a potential representation of how such a wider-scope model might become. However, this structure poses several challenges, for example having to consider potential family contacts without double counting and being able to separate potential contacts that have children in other participating schools from those who do not have them, and also from contacts that do not have children in schools. Moreover, with this framework we also would need to extend the boundaries of the model posing additional difficulties that are not necessary for the aim of the paper that it is just to qualitatively show the potential of engagement of schools in sustainability projects to diffuse sustainability culture.

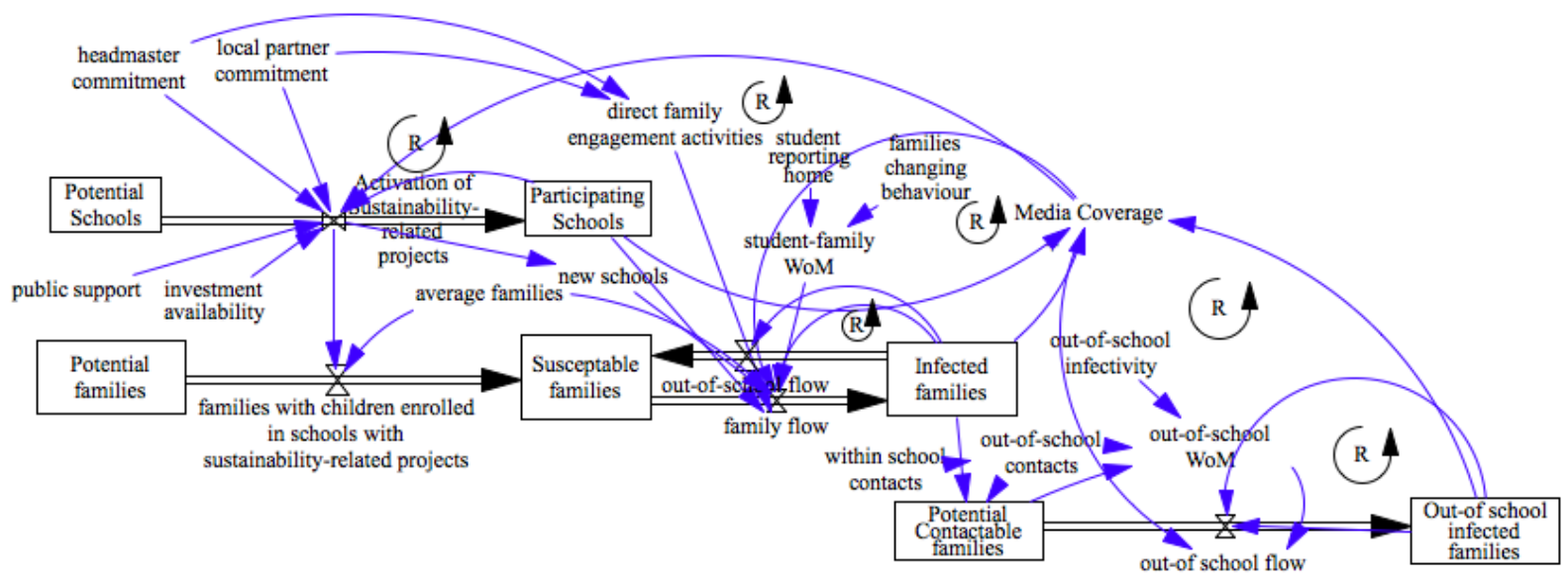

Figure 4.3. Potential extended model.

As specified in Section 3, given the limited availability of data we simulate not only one model, but a set of 1,000 runs characterised by different combinations of parameter values in order to test and show the sensitivity of the results to the data. More in detail, we performed repeated simulations in which model parameters are changed for each simulation drawing from probability distributions. The method adopted is the Vensim ${ }^{\mathrm{TM}}$ PLE Plus built-in multivariate search, whereby all parameters are changed together.

Results concerning the stocks of families changing over time are shown in Figure 4.4. Given the number of simulations we report results in the form of percentiles. The graphs show that the 'sustainable behaviour' epidemic does spread by engaging with schools and universities. Indeed, several families do change their behaviour after their children have been involved in school sustainability projects.

What also emerges from the model is a certain readiness at being informed and engaged. Indeed, even with the use of conservative hypothesis on several parameters, like the availability of headmaster commitment, local partner commitments, public support and investment availability, results concerning potential families (related to the school activation process) are heavy left loaded, meaning that under certain conditions they can all become susceptible to the epidemic. Results on susceptible families show how this stock tends to get empty, indeed, in most cases much of the flow coming from the potential family stock moves to the infected one, thus diffusing the behavioural change. Results on the levels of infected 
families, although emerging from potential and only indicative scenarios of a simple model, highlight how changes in behaviour following an enlargement of decision assessment models can spread.
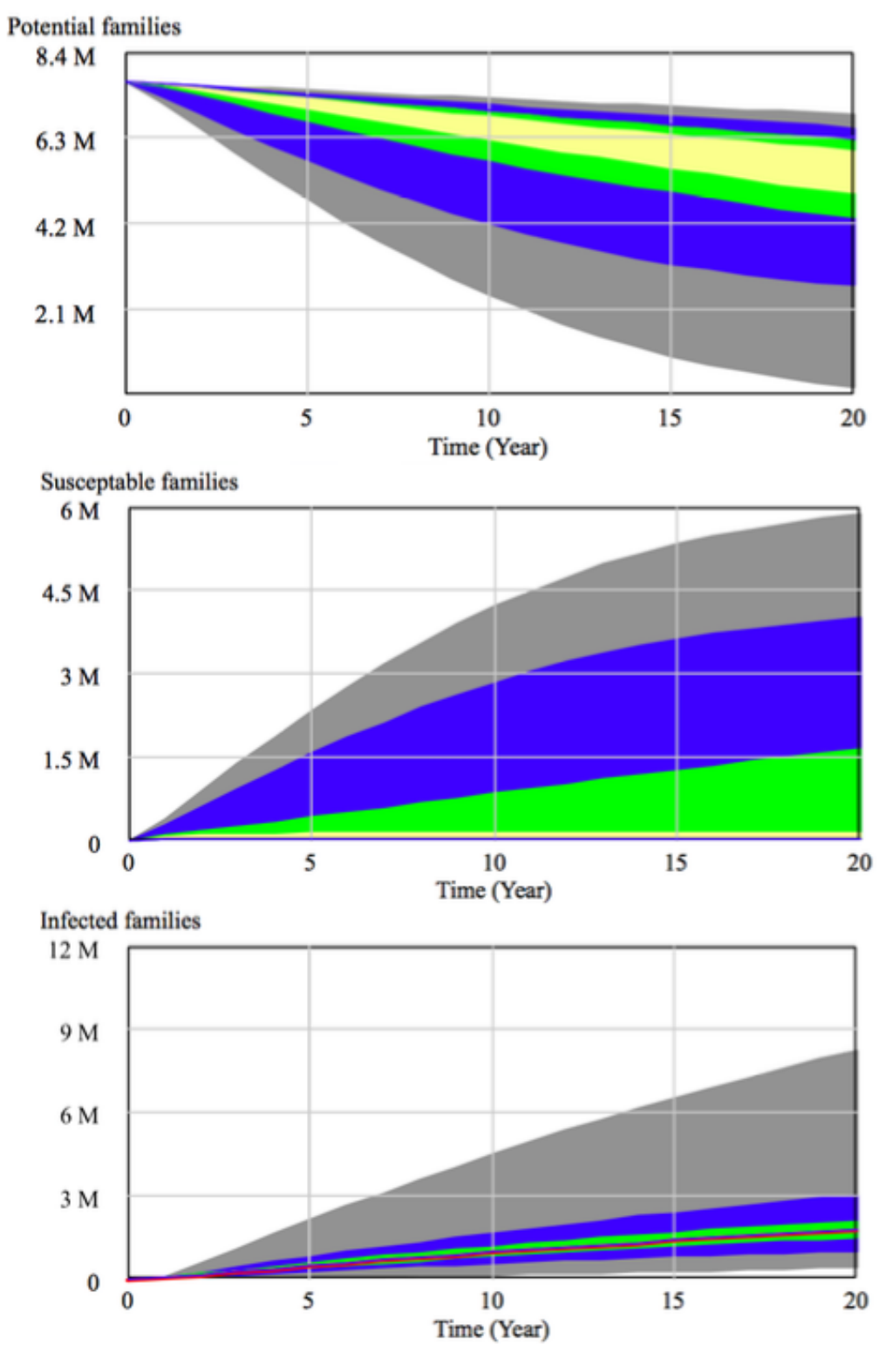

$50 \% \quad 75 \% \quad 795 \% \square$

Figure 4.4. Simulation results.

\section{Conclusions and limitations}

Results indicate that, given the chosen model parameters and functional forms, the 'sustainability epidemic' does spread. Indeed, schools are valid vectors of knowledge, sensitivity diffusion and may also promote behavioural changes. Indeed, even with quite conservative assumptions about the number of contacts and infectivity levels between students and families, all simulations indicate that in ten years several thousand or millions 
(depending of the local or national scale of the model) of households can be affected by a sustainability 'sensitisation' able to affect behaviour, at least for what concerns electricity use and food choices, that are the two domains investigated during data collection.

This can generate important societal benefits and go in the direction of actively engaging citizens/consumer in the solutions to current societal challenges, like global climate change. Indeed, being able to engage with large numbers of citizens/consumer with various sociodemographic conditions and geographically distributed across all the national territory could allow policy makers to consider individual behaviours within mitigation policies.

Even though the model proposed is very simple, it still allows to capture some of the interaction mechanisms among people connected with schools and (g)local communities; and it highlights that schools can be engaged on an ambitious ethical goal of sustainability culture diffusion, that can turn into more conscious and, possibly, more 'sustainable' choices and practices. The choice of the modelling tools allows to consider non-linear relationships, feedback loops and time lags that are important features of diffusion of information, culture and behaviour in the real world. Moreover, the project aims at diffusing sustainability culture and systemic perspectives that need to become widespread to be able to try to trigger the transition towards a more sustainable society.

The above discussed results need to be evaluated carefully in light of the limitations of the study. One of the limitations of this paper is linked to the fact that the proposed results depend on the choice of the parameters used to define the strength of the relationships between the variables. In particular, currently there are few data available on the strength of contagion between people on sustainability matters. Given the possibility of working with schools, we were able to collect data some useful data and adopted a Monte Carlo approach to evaluate the sensitivity of the model to variability in the parameter values. Though, even if data collected ad hoc is used to calibrate the model parameters, there is currently no available data on actual behaviours to validate the model. Moreover, the data collected refers to a sample that is limited in size and geographical scope. However, within the project, data is being collected on a yearly basis and each participant will be monitored for at least 5 consecutive years. Furthermore, the model formulation includes a set of exogenous parameters that could instead be made endogenous (Richardson, 2011). In any case, this is just a first preliminary study to show the potentials of engaging with schools on sustainability topic given their ability to reach very diverse segments of the population in terms of income and education levels, but also geographically. It is not by all means intended to be an accurate or exhaustive model, nor one to be used for actual prediction.

Future work will develop further the conceptual model and add more detail to the simulation model. For example, parameters can be, for example, differentiated by socio-demographic variables, as suggested in the literature, and by type of school. In fact, it is likely that depending on the age group of the students the involvement of families will change. Moreover, the model in its current form is not able to provide insights as to whether changes (or resistance to change) in the economic system may interact with the rise in awareness and its effects on behaviour. The boundaries of this model are limited to the diffusion of (stated) enrichments of individual decision-making models to include sustainability variables in the choice for food or energy choices.

Future work will also analyse jointly the effect of different events on citizen/consumer behaviour looking at media coverage of international environmental events or declarations by 
opinion leaders (COP meetings, Pope's Francis Encyclical, Greta Thunberg and Fridays for Future events, Donald Trump, and other prominent actors).

Future applications might also want to build a spatial model that can model the fact that imitation and 'social' pressure on schools, families/individuals and other actors may have a geographical distribution related to 'glocal' proximity.

\section{Reference list}

Agovino, M., Crociata, A., Quaglione, D., Sacco, P., and Sarra, A. (2017), “Good Taste Tastes Good. Cultural Capital as a Determinant of Organic Food Purchase by Italian Consumers: Evidence and Policy Implications", Ecological Economics, 141: 66-75.

Aguirre-Bielschowsky, I., Lawson, R., Stephenson, J., and Todd, S. (2018), “Kids and Kilowatts: Socialisation, Energy Efficiency, and Electricity Consumption in New Zealand", Energy Research \& Social Science, 44: 178-186.

Ajzen, I. (1991), “The Theory of Planned Behavior", Organizational Behavior and Human Decision Processes, 50 (2):179-211.

Ajzen, I. (2011), “The Theory of Planned Behaviour: Reactions and Reflections”, Psychology $\mathcal{E}$ Health, 26 (9): 1113-1127.

Alcott, H. (2011), "Social Norms and Energy Conservation", Journal of Public Economics 95 (910):1082-1095.

Arvola, A., Vassallo, M., Dean, M., Lampila, P., Saba, A., Lähteenmäki, L., and Shepherd, R. (2008), "Predicting Intentions to Purchase Organic Food: The Role of Affective and Moral Attitudes in the Theory of Planned Behaviour", Appetite, 50 (2): 443-454.

Bass, F.M. (1969), “A New Product Growth for Model Consumer Durables”, Management Science, 15 (5): 215-227.

Boccaletti, S. (2008), “Environmentally Responsible Food Choice”, in I. Ferrara and Serret Y. (eds), Household Behaviour and the Environment Reviewing the Evidence, 117-152, Paris: OECD.

Bremer, M.H., and López-Franco, R. (2006), "Sustainable Development: Ten Years of Experience at Itesm's Graduate Level", Journal of Cleaner Production, 14 (9-11): 952-957.

Daniels, S., Glorieux, I., Minnen, J., van Tienoven, T.P., and Weenas, D. (2015), “Convenience on the Menu? A Typological Conceptualization of Family Food Expenditures and FoodRelated Time Patterns", Social Science Research, 51: 205-218.

Darby, S. (2010), “Smart Metering: What Potential for Householder Engagement?", Building Research $\mathcal{E}$ Information, 38 (5): 442-457.

Darby, S. (2006), The Effectiveness of Feedback on Energy Consumption: A Review for DEFRA of the Literature on Metering, Billing and Direct Displays, Oxford: Oxford Environmental Change Institute.

De Groot, J., and Steg, L. (2007), “General Beliefs and the Theory of Planned Behavior: The Role of Environmental Concerns in the TPB", Journal of Applied Social Psychology, 37 (8): 1817-1836. 
Drichoutis, A.C., Lazaridis, P., and Nayga Jr., R.M. (2006), “Consumers' Use of Nutritional Labels: A Review of Research Studies and Issues", Academy of Marketing Science Review, 9 (9): 1-22.

D’Souza, C., Taghian, M., Lamb, P., and Peretiatko, R. (2007), “Green Decisions: Demographics and Consumer Understanding of Environmental Labels", International Journal of Consumer Studies, 31 (4): 371-376.

Eberlein, R.L., and Peterson, D.W. (1992), “Understanding Models with Vensim ${ }^{\mathrm{TM}}$, , European Journal of Operational Research, 59 (1): 216-219.

Eden, S., Bear, C., and Walker, G. (2008), “Understanding and (Dis)Trusting Food Assurance Schemes: Consumer Confidence and the 'Knowledge Fix'", Journal of Rural Studies, 24 (1): $1-14$.

European Commission (2014), Special Eurobarometer 416: Attitudes of European Citizens Towards the Environment, Brussels: European Commission, Directorate-General for Environment (DG ENV). Available online at http://ec.europa.eu/public_opinion/archives/ebs/ebs_416_en.pdf (last accessed: April 15, 2019).

Evans, D. (2011), “Consuming Conventions: Sustainable Consumption, Ecological Citizenship and the Worlds of Worth", Journal of Rural Studies, 27 (2): 109-115.

Forrester, J.W. (1961), Industrial Dynamics, Cambridge (MA): MIT Press.

Forrester, J.W. (2007), "System Dynamics: A Personal View of the First Fifty Years”, System Dynamics Review: The Journal of the System Dynamics Society, 23 (2-3): 345-358.

Gilg, A., Barr, S., and Ford., N. (2005), “Green Consumption or Sustainable Lifestyles? Identifying the Sustainable Consumer", Futures, 37 (6): 481-504.

Hartmann, P., and Apaolaza-Ibáñez, V. (2012), “Consumer Attitude and Purchase Intention toward Green Energy Brands: The Roles of Psychological Benefits and Environmental Concern", Journal of Business Research, 65 (9): 1254-1263.

Kühtz, S. (2007), “Adoption of Sustainable Development Schemes and Behaviours in Italy: Barriers and Solutions - What Can Educators Do?", International Journal of Sustainability in Higher Education, 8 (2): 155-169.

Kurz, T., Gardner, B., Verplanken, B., and Abraham, C. (2015), “Habitual Behaviors or Patterns of Practice? Explaining and Changing Repetitive Climate-Relevant Actions", Wiley Interdisciplinary Reviews: Climate Change, 6 (1): 113-128.

Lozano, R., Lukman, R., Lozano, F.J., Huisingh, D., and Lambrechts, W. (2013), “Declarations for Sustainability in Higher Education: Becoming Better Leaders, through Addressing the University System", Journal of Cleaner Production, 48: 10-19.

Meadows, D.H. (2008), Thinking in Systems: A Primer, Vermont: Chelsea Green Publishing.

Mella, P. (2012), Systems Thinking: Intelligence in Action, Heidelberg: Springer Science \& Business Media.

Murray, J.D. (2002), Mathematical Biology: An Introduction (3 ${ }^{\text {rd }}$ edn.), New York (NY): SpringerVerlag. 
Pedersen, E.R., and Neergaard, P. (2006a), "Caveat Emptor - Let the Buyer Beware! Environmental Labelling and the Limitations of 'Green' Consumerism", Business Strategy and the Environment, 15 (1): 15-29.

Pongiglione, F., and Cherlet, J. (2015), "The Social and Behavioral Dimensions of Climate Change: Fundamental but Disregarded?", Journal for General Philosophy of Science, 46 (2): 383-391.

Ricci, E.C. (2013), "Smart-Grids and Climate Change. Consumer Adoption of Smart Energy Behaviour: A System Dynamics Approach to Evaluate the Mitigation Potential", Climate Change and Sustainable Development Series, Nota di Lavoro, Fondazione Eni Enrico Mattei.

Ricci, E.C., and Banterle, A. (2018), “The Effects of Expo Milano 2015 on Consumer Food Choices", Economia Agro-Alimentare, 20 (2): 233-244.

Richardson, G.P. (2011), "Reflections on the Foundations of System Dynamics", System Dynamics Review, 27 (3): 219-243.

Rieckmann, M. (2012), “Future-Oriented Higher Education: Which Key Competencies Should Be Fostered through University Teaching and Learning?", Futures, 44 (2): 127-135.

Sibbel, A. (2009), "Pathways Towards Sustainability through Higher Education", International Journal of Sustainability in Higher Education, 10 (1): 68-82.

Steg, L., and Vlek, C. (2009), "Encouraging Pro-Environmental Behaviour: An Integrative Review and Research Agenda", Journal of Environmental Psychology, 29 (3): 309-317.

Sterman, J. (2000), Business Dynamics: Systems Thinking and Modeling for a Complex World, Boston (MA): Irwin/McGraw Hill.

Sunstein, C.R. (2013), "Behavioral Economics, Consumption, and Environmental Protection”, SSRN Electronic Journal: 1-29. Available online at http://www.ssrn.com/abstract=2296015 (last accessed: April 15, 2019).

Testa, F., Iraldo, F., Vaccari, A., and Ferrari, E. (2015), "Why Eco-Labels Can Be Effective Marketing Tools: Evidence from a Study on Italian Consumers", Business Strategy and the Environment, 24 (4): 252-265.

Thaler, R., and Sunstein, C. (2008), Nudge: The Gentle Power of Choice Architecture, New Haven (CT): Yale University Press.

Urban, J., and Ščasný, M. (2012), “Exploring Domestic Energy-Saving: The Role of Environmental Concern and Background Variables", Energy Policy, 47: 69-80.

Vermeir, I., and Verbeke, W. (2006), "Sustainable Food Consumption: Exploring the Consumer 'Attitude-Behavioral Intention' Gap", Journal of Agricultural and Environmental Ethics, 19 (2): 169-194.

Verplanken, B., and Aarts, H. (1999), “Habit, Attitude, and Planned Behaviour: Is Habit an Empty Construct or an Interesting Case of Goal-Directed Automaticity?", European Review of Social Psychology, 10 (1): 101-134. 